\title{
SPECTRAL THEORY OF DAMPED QUANTUM CHAOTIC SYSTEMS
}

\author{
STÉPHANE NONNENMACHER
}

\begin{abstract}
We investigate the spectral distribution of the damped wave equation on a compact Riemannian manifold, especially in the case of a metric of negative curvature, for which the geodesic flow is Anosov. The main application is to obtain conditions (in terms of the geodesic flow on $X$ and the damping function) for which the energy of the waves decays exponentially fast, at least for smooth enough initial data. We review various estimates for the high frequency spectrum in terms of dynamically defined quantities, like the value distribution of the timeaveraged damping. We also present a new condition for a spectral gap, depending on the set of minimally damped trajectories.
\end{abstract}

\section{INTRODUCTION}

1.1. Spectrum of the damped wave equation. Given a Riemannian manifold $(X, g)$ and a damping function $a \in C^{\infty}\left(X, \mathbb{R}_{+}\right)$, we are interested in the solutions of the damped wave equation (DWE)

$$
\left(\partial_{t}^{2}-\Delta+2 a(x) \partial_{t}\right) v(x, t)=0, \quad v(x, 0)=v_{0}, \partial_{t} v(x, 0)=v_{1} .
$$

The natural setting is to take intial data $\left(v_{0}, v_{1}\right)$ in the space $\mathcal{H} \stackrel{\text { def }}{=} H^{1}(X) \times L^{2}(X)$. This equation is equivalent with the system

$$
\left(i \partial_{t}+\mathcal{A}\right) \boldsymbol{v}(t)=0, \quad \mathcal{A} \stackrel{\text { def }}{=}\left(\begin{array}{cc}
0 & I \\
-\Delta & -2 i a
\end{array}\right), \quad \boldsymbol{v}(0) \in \mathcal{H}
$$

with the correspondence $\boldsymbol{v}(t)=(v(t), i \partial v(t))$. $\mathcal{A}$ generates a strongly continuous semigroup on $\mathcal{H}$, so the solution to (1.1]1.2) reads

$$
\boldsymbol{v}(t)=e^{-i t \mathcal{A}} \boldsymbol{v}(0) .
$$

We will always assume that the damping is nontrivial, $a \not \equiv 0$. Apart from the constant solution $\boldsymbol{v}(t)=(1,0)$, all solutions then decay. Physically, this is expressed by the fact that the energy of the waves,

$$
E(v(t))=\frac{1}{2}\left(\|\nabla v(t)\|^{2}+\left\|\partial_{t} v(t)\right\|^{2}\right),
$$

will decay to zero when $t \rightarrow \infty$ for any $\boldsymbol{v}(0) \in \mathcal{H}$. In some sense, the waves are stabilized by the damping.

To analyze this decay, it it natural to try to expand the solution in terms of the spectrum of $\mathcal{A}$. This spectrum is discrete, consisting of countably many complex eigenvalues $\left\{\tau_{n}\right\}$ with $\operatorname{Re} \tau_{n} \rightarrow \pm \infty$. It can be obtained by solving the generalized eigenvalue equation

$$
\left(-\Delta-\tau^{2}-2 i a \tau\right) u=0 .
$$

This work has been partially supported by the grant ANR-09-JCJC-0099-01 of the Agence Nationale de la Recherche. 


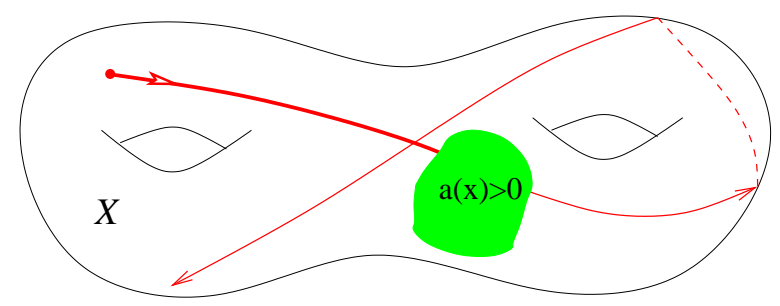

Figure 1.1. A damped geodesic.

The following properties are easily shown $\left(a_{\min } \stackrel{\text { def }}{=} \min _{x \in X} a(x)\right.$, similarly for $\left.a_{\max }\right)$.

Proposition 1. Leb93] All eigenvalues except $\tau_{0}=0$ satisfy $\operatorname{Im} \tau_{n}<0$.

If $\operatorname{Re} \tau_{n} \neq 0$ then $-\operatorname{Im} \tau_{n} \in\left[a_{\min }, a_{\max }\right]$.

The spectrum is symmetric w.r.to the imaginary axis.

To each eigenvalue $\tau_{n}$ corresponds a quasi-stationary mode $u_{n}(x)$, an eigenstate $\boldsymbol{u}_{n}=\left(u_{n}, \tau_{n} u_{n}\right)$ of $\mathcal{A}$ reads, and a solution $v_{n}(t, x)=e^{-i t \tau_{n}} u_{n}(x)$ of the DWE. Hence $\operatorname{Im} \tau_{n}$ represents the quantum decay rate of the mode $u_{n}$.

If $a_{\min }>0$, then the energy decays exponentially, uniformly for initial data $\boldsymbol{v}(0) \in$ $\mathcal{H}$. Precisely, there exists $\gamma>0, C>0$ such that

$$
E(\boldsymbol{v}(t)) \leq C e^{-2 \gamma t} E(\boldsymbol{v}(0)), \quad \forall \boldsymbol{v}(0) \in \mathcal{H}, \quad \forall t \geq 0 .
$$

1.2. The Geometric Control Condition. The condition $a_{\min }>0$ is not necessary to ensure such a uniform exponential decay. Since all eigenvalues except $\tau_{0}=0$ satisfy $\operatorname{Im} \tau_{n}<0$, for any $C>0$ the subspace $\mathcal{H}_{C} \subset \mathcal{H}$ spanned by the eigenstates $\left\{\boldsymbol{u}_{n},\left|\operatorname{Re} \tau_{n}\right| \leq C\right\}$ is finite dimensional, and for any initial data $\boldsymbol{v}(0) \subset \mathcal{H}_{C}$ the energy will decay exponentially. Hence, the failure of exponential decay can only come from the behaviour of waves at high frequency. In this high frequency limit, a natural connection can be made with the classical ray dynamics on $X$, equivalently the geodesic flow $\Phi^{t}$ on the unit cotangent bundle $S^{*} X$ (see $\$ 1.4$ ). Using this connection, Rauch and Taylor RauTay75 showed that the uniform exponential decay (1.6) is equivalent with the Geometric Control Condition (GCC), which states that every geodesic meets the damping region $\{x \in X, a(x)>0\}$ (due to the compactness of $S^{*} X$ each geodesic does it within some time $\left.T_{0}>0\right)$.

This condition can be expressed in terms of the time averages of the damping, namely the functions

$$
\langle a\rangle_{t}(\rho)=\frac{1}{t} \int_{0}^{t} a \circ \Phi^{s}(\rho) d s, \quad \rho \in T^{*} X, t>0 .
$$

GCC is equivalent to the fact that, for $t>0$ large enough (say, $t>2 T_{0}$ ), the function $\langle a\rangle_{t}$ is strictly positive on $S^{*} X$. Lebeau Leb93] generalized this result to the case of manifolds with boundaries. He also showed that the optimal decay rate $\gamma$ is given by $\min \left(G, a_{-}\right)$, where $G=\inf \left\{-\operatorname{Im} \tau_{n}, \tau_{n} \neq 0\right\}$ is the spectral gap, while

$$
a_{-} \stackrel{\text { def }}{=} \lim _{t \rightarrow \infty} \min _{S^{*} X}\langle a\rangle_{t}
$$

is the minimal asymptotic damping. We will explain the relevance of $a_{-}$in $§ 2.2$.

Koch and Tataru [KoTa94] studied the same question in a more general context (case of manifolds with boundaries, and of a damping taking place both in the 
"bulk" and on the boundary). They showed that the averages $\langle a\rangle_{t}$ govern the decay of the semigroup, up to a compact subspace.

Theorem 2. [KoTa94, Thm 2] For each $\epsilon>0$ and each $t>0$ there exists a subspace $\mathcal{H}_{\epsilon, t} \subset \mathcal{H}$ of finite codimension such that

$$
\left\|e^{-i t \mathcal{A}}\right\|_{\mathcal{H}_{\epsilon, t} \rightarrow \mathcal{H}} \leq \exp \left\{-t \min _{S^{*} X}\langle a\rangle_{t}\right\}+\epsilon .
$$

For any subspace $\mathcal{H}_{1} \subset \mathcal{H}$ of finite codimension,

$$
\exp \left\{-t \min _{S^{*} X}\langle a\rangle_{t}\right\} \leq\left\|e^{-i t \mathcal{A}}\right\|_{\mathcal{H}_{1} \rightarrow \mathcal{H}} \cdot
$$

A consequence of this result is the characterization of the Fredholm spectrum of the semigroup. In the present situation of damped waves on a compact $X$ withough boundary, their result states 1 that this Fredholm spectrum is given by the annulus $\left\{z \in \mathbb{C}, e^{-t a_{+}} \leq|z| \leq e^{-t a_{-}}\right\}$.

Notice that it is possible to have a positive gap $G>0$ and at the same time $a_{-}=0$ (failure of the GCC), see e.g. an example in [Ren94]. This reflects the fact that the spectrum of the semigroup $e^{-i t \mathcal{A}}$ is not controlled by the spectrum of generator $\mathcal{A}$, a frequent problem for nonnormal generators like $\mathcal{A}$.

1.3. Beyond Geometric control: a few cases of uniform energy decay for regular data. If GCC fails, one can construct initial data $\boldsymbol{v}(0) \in \mathcal{H}$ such that $E(\boldsymbol{v}(t))$ decays arbitrarily slowly [Leb93]. Yet, it is possible to show that more regular data, say in some Sobolev space $\mathcal{H}^{s}=H^{s+1} \times H^{s}, s>0$, decay in some uniform way. In the most general situation, Lebeau showed a logarithmic decay of the energy [Leb93, Thm 1],

$$
E(\boldsymbol{v}(t))^{1 / 2} \leq C_{s} \frac{\log [3+\log (3+t)]}{\log (3+t)^{s}}\|\boldsymbol{v}\|_{\mathcal{H}^{s}}, \quad \forall \boldsymbol{v}=\boldsymbol{v}(0) \in \mathcal{H}^{s}, \forall t \geq 0 .
$$

The proof uses Carleman estimates, which imply some control of the resolvent $(\tau-\mathcal{A})^{-1}$ in a strip $\{\operatorname{Re} \tau \geq C,|\operatorname{Im} \tau| \leq f(\operatorname{Re} \tau)\}$ for some function $f(r)$ which decays exponentially as $r \rightarrow \infty$. By interpolation, proving such an estimate for some $s_{0}>0$ implies a similar estimate for all $s>0$.

In specific dynamical situations, one can show resolvent estimates in a larger strip, e.g. for some function $f(r)$ decaying algebraically: one then gets an algebraic decay $\mathcal{O}\left(t^{-\gamma_{s}}\|\boldsymbol{v}\|_{\mathcal{H}^{s}}\right)$ for the energy of regular data. Burq and Hitrik showed that this is the case for damped waves in the (chaotic) stadium billiard, if the damping function vanishes only in some part of the central rectangle, so that the set of undamped trajectories,

$$
K \stackrel{\text { def }}{=}\left\{\rho \in S^{*} X, \forall t \in \mathbb{R}, a\left(\Phi^{t}(\rho)\right)=0\right\},
$$

consists in a collection of (neutrally stable) "bouncing ball" trajectories [BuHi07].

Christianson studied the case where the set $K$ consists in a single hyperbolic closed geodesic: the energy decay is bounded by a stretched exponential $\mathcal{O}\left(e^{-\gamma_{s} t^{1 / 2}}\|\boldsymbol{v}\|_{\mathcal{H}^{s}}\right)$ [Chris07, Thm 5'] Chris11]. The hyperbolicity of the geodesic induces a strong dispersion of the waves, responsible for this fast decay.

\footnotetext{
${ }^{1}$ under the condition that, for any $T>0$, the set of $T$-periodic geodesics has measure zero.
} 
In 93.3 and 3.4 we will present situations, with $(X, g)$ a manifold of negative curvature, for which the energy of regular data decays exponentially:

$$
E(\boldsymbol{v}(t))^{1 / 2} \leq C_{s} e^{-\gamma_{s} t}\|\boldsymbol{v}\|_{\mathcal{H}^{s}}, \quad \forall \boldsymbol{v} \in \mathcal{H}^{s}, \forall t \geq 0 .
$$

The proof proceeds by controlling the resolvent $(\tau-\mathcal{A})^{-1}$ in a strip $\{\operatorname{Re} \tau>C,|\operatorname{Im} \tau| \leq c\}$, and then uses standard arguments [Chris09]. Here as well, the resolvent bounds are based on certain hyperbolic dispersion estimates. See the Corollaries 16 and 19 for the precise dynamical conditions, which involve the interplay between the flow and the damping function.

\subsection{High frequency limit - semiclassical formulation and generalization.}

As explained above, the decay of the energy is mainly governed by the high frequencies $\operatorname{Re} \tau \gg 1$. A semiclassical formulation of the problem was used in Sjo00, allowing to use $\hbar$-pseudodifferential techniques. Take an effective Planck's "constant" $0<\hbar \ll 1$. In order to study the frequencies $\operatorname{Re} \tau=\hbar^{-1}+\mathcal{O}(1)$, we perform the rescaling

$$
\tau=\frac{\sqrt{2 z}}{\hbar} \quad \text { with } z \text { in the disk } D(1 / 2, C \hbar)
$$

The equation (1.5) becomes

$$
(P(\hbar, z)-z) u=0, \quad P(\hbar, z)=-\frac{\hbar^{2} \Delta}{2}-i \hbar \sqrt{2 z} a=-\frac{\hbar^{2} \Delta}{2}-i \hbar a+\mathcal{O}\left(\hbar^{2}\right) .
$$

More generally, we consider operators of the type

$$
P(\hbar, z)=-\frac{\hbar^{2} \Delta}{2}+i \hbar \mathrm{Op}_{\hbar}\left(q_{z}\right)
$$

where the symbol $q_{z} \in S^{0}\left(T^{*} X\right)$ is now a function on the phase space $T^{*} X$, and depends holomorphically on $z \in D(1 / 2, C \hbar)$, with $q_{z}=q_{1 / 2}+\mathcal{O}(\hbar)$ [Sjo00]. With a slight abuse, will call $q=q_{1 / 2}(x, \xi)$ "the damping function" (a damping effectively occurs only at phase space points where $q(x, \xi)<0$, while positive values of $q$ rather correspond to a "gain" or "pumping").

In this framework, we are interested in the semiclassical distribution of the generalized eigenvalues $z_{n}(\hbar)$ of the operator $P(\hbar, z)$ in the disk $D(1 / 2, C \hbar)$. Because we will specify this spectrum at the scale $\hbar$, we may for convenience drop the term $\mathcal{O}\left(\hbar^{2}\right)$ in (1.13), and rather study the spectrum of the $z$-independent operator

$$
P(\hbar)=P_{0}(\hbar)+i \hbar \mathrm{Op}_{\hbar}(q), \quad P_{0}(\hbar) \stackrel{\text { def }}{=}-\frac{\hbar^{2} \Delta}{2} .
$$

The principal symbol of this operator is $p_{0}(x, \xi)=\frac{|\xi|^{2}}{2}$, it generates the geodesic flow $\Phi^{t}=\exp \left(t H_{p_{0}}\right)$ on $T^{*} X$, with unit speed on the energy shell $p_{0}^{-1}(1 / 2)=S^{*} X$.

\footnotetext{
${ }^{2}$ For any $k \in \mathbb{R}$, the symbol class $S^{k}\left(T^{*} X\right)$ denotes the functions $a(x, \xi ; \hbar)$ satisfying the following bounds: for any multi-indices $\alpha, \beta \in \mathbb{N}^{d}$,

$$
\left|\partial_{x}^{\alpha} \partial_{\xi}^{\beta} a(x, \xi ; \hbar)\right| \leq C_{\alpha, \beta}(1+|\xi|)^{k-|\beta|} \quad \text { uniformly for } \hbar \in(0,1),
$$

By $\mathrm{Op}_{\hbar}(q)$ we refer to a semiclassical quantization on $X$, with the property that $\mathrm{Op}_{\hbar}(q)$ is symmetric if $q$ is real (see e.g [EvZw App. E]). The operators $\mathrm{Op}_{\hbar}(q)$ form the class $\Psi^{k}(X)$ of pseudodifferential operators (PDO).
} 


\section{Semiclassical spectral distribution of $P(\hbar)$. General Results}

The results we present in this section hold for arbitrary Riemannian manifolds $(X, g)$. We skip the proofs.

2.1. Weyl law for the real parts. In the semiclassical limit, the number of eigenvalues of the selfadjoint operator $P_{0}(\hbar)=-\frac{\hbar^{2} \Delta}{2}$ in the interval $\left[\frac{1}{2} \pm \epsilon\right] \stackrel{\text { def }}{=}$ $\left[\frac{1}{2}-\epsilon, \frac{1}{2}+\epsilon\right]$ is approximately given by Weyl's law. When applying the perturbation $i \hbar \mathrm{Op}_{\hbar}(q)=\mathcal{O}(\hbar)$, it seems natural to expect that most eigenvalues of $P_{0}(\hbar)$ in this interval will remain inside a (complex) neighbourhood $\left[\frac{1}{2} \pm \epsilon\right]+\mathcal{O}(\hbar)$. One can indeed show that the "macroscopic" spectral distribution is given by the same Weyl's law as in the undamped case.

Theorem 3. MarMat84, Sjo00 For any $\epsilon>0$ small enough (possibly depening on $\hbar)$, the number of eigenvalues of $P(\hbar)$ in the strip $\mathcal{S}_{\epsilon}=\left\{\frac{1}{2}-\epsilon \leq \operatorname{Re} z \leq \frac{1}{2}+\epsilon\right\}$ is semiclassically given by

$$
\#\left\{\operatorname{Spec} P(\hbar) \cap \mathcal{S}_{\epsilon}\right\}=(2 \pi \hbar)^{-d} \operatorname{Vol}\left\{\rho \in T^{*} X,\left|p_{0}(\rho)-1 / 2\right| \leq \epsilon\right\}+\mathcal{O}\left(\hbar^{-d+1}\right) .
$$

Here Vol corresponds to the symplectic volume in $T^{*} X$. This estimate implies that, for $C>0$ large enough,

$$
\#\{\operatorname{Spec} P(\hbar) \cap D(1 / 2, C \hbar)\} \asymp \hbar^{-d+1} .
$$

This result is actually not so easy to prove. It uses some subtle analytic Fredholm theory in order to relate the spectrum of $P(\hbar)$ with that of a simpler "comparison operator".

2.2. Restrictions for the imaginary parts (quantum decay rates). Eventhough the operator $P(\hbar)$ was inspired by the wave equation, it is convenient for us to consider it instead as the generator of a Schrödinger equation

$$
i \hbar \partial_{t} v-P(\hbar) v=0 .
$$

Any eigenvalue/vector $\left(z_{n}, u_{n}\right)$ of $P(\hbar)$ is thus associated with a solution $v_{n}(x, t)=$ $e^{-i z_{n} t / \hbar} u_{n}(x)$ of this equation, and we will also call $\operatorname{Im} z_{n} / \hbar$ the quantum decay rat 3 associated with $u_{n}$.

First we remark that an eigenmode $u_{n}$ associated to an eigenvalue $z_{n} \in D(1 / 2, C \hbar)$ is semiclassically microlocalized on $p_{0}^{-1}(1 / 2)=S^{*} X$. To state this property we need to introduce energy cutoffs.

Definition 4. An energy cutoff will be function $\chi \in C_{c}^{\infty}([1 / 2 \pm \delta],[0,1])$ for some $\delta>0$, and satisfies $\chi(s)=1$ near $s=1 / 2$. The cutoff $\chi_{0}$ is said to be embedded in $\chi_{1}$, and we note $\chi_{1} \succ \chi_{0}$, iff $\chi_{1} \equiv 1$ near supp $\chi_{0}$. From the cutoff $\chi$ we construct the (pseudodifferential) cutoff operator $\chi\left(P_{0}(\hbar)\right) \in \Psi^{-\infty}(X)$.

Proposition 5. Take an energy cutoff $\chi$. Let $u=u(\hbar)$ be an eigenstate of $P(\hbar)$ with eigenvalue $z(\hbar) \in D(1 / 2, C \hbar)$. Then,

$$
\left\|\left(I-\chi\left(P_{0}\right)\right) u\right\|=\mathcal{O}\left(\hbar^{\infty}\right)\|u\| .
$$

This localization property explains why only the neighbourhood of $S^{*} X$ will be important for us.

\footnotetext{
${ }^{3}$ From (1.11) we have $\operatorname{Im} z_{n} / \hbar=\operatorname{Im} \tau_{n}+\mathcal{O}(\hbar)$, so this denomination is (approximately) consistent with the one of $\$ 1.1$.
} 
2.2.1. A factorization of the Schrödinger propagator. An easy way to realize how the skew-adjoint subprincipal term $i \hbar \mathrm{Op}_{\hbar}(q)$ implies a "damping" is to analyze the propagator for the Schrödinger equation (2.2). The following Proposition adapts a more general result due to Rauch and Taylor RauTay75.

Proposition 6. Assume $q \in C_{c}^{\infty}\left(T^{*} X\right)$, and take $P(\hbar)$ as in 1.14). For any fixed $t \in \mathbb{R}$, decompose the Schrödinger propagator $V^{t}=e^{-i t P / \hbar}$ into

$$
V^{t}=U^{t} B(t) \text {, where } U^{t}=e^{-i t P_{0} / \hbar} \text { is the undamped propagator. }
$$

The operator $B(t)$ is a PDO in $\Psi^{0}(X)$ of principal symbol

$$
b(t, \rho)=\exp \left\{\int_{0}^{t} q \circ \Phi^{s}(\rho) d s\right\}=\exp \left\{t\langle q\rangle_{t}(\rho)\right\},
$$

where the time averaged damping $\langle q\rangle_{t}$ generalizes (1.7).

The factor $b(t, \rho)$ is the accumulated damping along the orbit $\left\{\Phi^{s}(\rho), 0 \leq s \leq t\right\}$. If one starts from a Gaussian wavepacket $u_{0}$ microlocalized at a point $\rho$, then the state $V^{t} u_{0}$ is a wavepacket microlocalized at $\Phi^{t}(\rho)$, and the above statement shows that the $L^{2}$ norms are related through this factor:

$$
\left\|u_{t}\right\|=\left\|u_{0}\right\|(b(t, \rho)+\mathcal{O}(\hbar)) .
$$

Applying an energy cutoff allows to extend the factorization (2.3) damping functions $q \in S^{0}\left(T^{*} X\right)$ of noncompact support.

Lemma 7. Consider $P(\hbar)$ with a damping function $q \in S^{0}\left(T^{*} X\right)$. Take two embedded cutoffs $\chi_{1} \succ \chi_{0}$ supported in $[1 / 2 \pm \delta]$, and the truncated damping $\tilde{q} \stackrel{\text { def }}{=} \chi_{1}\left(p_{0}\right) q$.

Then, for any $t \geq 0$ fixed, one has

$$
V^{t} \chi_{0}\left(P_{0}\right)=\tilde{V}^{t} \chi_{0}\left(P_{0}\right)+\mathcal{O}_{L^{2} \rightarrow L^{2}}\left(\hbar^{\infty}\right),
$$

where $\tilde{V}^{t}$ is the propagator corresponding to $\tilde{P}=P_{0}+i \hbar \mathrm{Op}_{\hbar}(\tilde{q})$.

This identity uses the fact that the propagation $V^{t}$ does not modify the energy localization properties.

2.2.2. From propagator factorization to a resolvent bound. We can now easily obtain a first constraint on the quantum decay rates. Applying Proposition 6 to the function $\tilde{q}=\chi_{1}\left(p_{0}\right) q$ of Lemma 7, we get for any fixed $t \geq 0$ :

$$
\begin{aligned}
\left\|V^{t} \chi_{0}\left(P_{0}\right)\right\|_{L^{2} \rightarrow L^{2}} & =\left\|\tilde{B}(t) \chi_{0}\left(P_{0}\right)\right\|_{L^{2} \rightarrow L^{2}}+\mathcal{O}\left(\hbar^{\infty}\right) \\
& =\max _{T^{*} X} \tilde{b}(t) \chi_{0}\left(p_{0}\right)+\mathcal{O}_{t}(\hbar) \\
& \leq \exp \left\{t \max _{\operatorname{supp} \chi_{0} \circ p_{0}}\langle\tilde{q}\rangle_{t}\right\}+\mathcal{O}_{t}(\hbar) .
\end{aligned}
$$

On the second line we used the sharp Gårding (in)equality for the operator $\tilde{B}(t) \in$ $\Psi^{0}(X)$. The maximum $\max _{p_{0}^{-1}([1 / 2 \pm \delta])}\langle\tilde{q}\rangle_{t}$ decreases when $t \rightarrow \infty$ or when $\delta \rightarrow 0$, and converges to the asymptotic maximum on $S^{*} X$,

$$
q_{+} \stackrel{\text { def }}{=} \lim _{t \rightarrow \infty} \max _{\rho \in S^{*} X}\langle q\rangle_{t}(\rho) .
$$

One similarly defines an asymptotic minimum $q_{-}$. We then get the following norm bounds for the propagator. 
Proposition 8. Fix $\epsilon>0$. If the energy cutoff $\chi_{0}$ has a small enough support, and $T_{\epsilon}>0$ is large enough, then for any (fixed) $t \geq T_{\epsilon}$ and any small enough $\hbar>0$, the following bounds holds:

$$
\left\|V^{t} \chi_{0}\left(P_{0}\right)\right\|_{L^{2} \rightarrow L^{2}} \leq e^{\left(q_{+}+\epsilon\right) t}, \quad\left\|V^{-t} \chi_{0}\left(P_{0}\right)\right\|_{L^{2} \rightarrow L^{2}} \leq e^{\left(-q_{-}+\epsilon\right) t} .
$$

From there one easily obtains the following resolvent and spectral bounds.

Theorem 9. Leb93, Sjo00 Take any $\epsilon>0$. Then, there exists $\hbar_{\epsilon}, C_{\epsilon}>0$ such that for $\hbar<\hbar_{\epsilon}$, the following resolvent estimate holds:

$$
\forall z \in D(1 / 2, C \hbar) \backslash\left\{z, \operatorname{Im} z / \hbar \in\left[q_{-}-\epsilon, q_{+}+\epsilon\right]\right\}, \quad\left\|(P(\hbar)-z)^{-1}\right\| \leq \frac{C_{\epsilon}}{\hbar}
$$

As a consequence, for $\hbar<\hbar_{\epsilon}$ all eigenvalues $z_{n} \in D(1 / 2, C \hbar)$ of $P(\hbar)$ satisfy

$$
\frac{\operatorname{Im} z_{n}}{\hbar} \in\left[q_{-}-\epsilon, q_{+}+\epsilon\right] .
$$

In the case of the damped wave equation $(q(x, \xi)=-a(x))$ and in a situation of geometric control $\left(a_{-}=-q_{+}>0\right)$, the resolvent estimate (2.8) can be used to show the uniform exponential energy decay (1.6) Hit03.

2.3. Questions on the spectral distribution. So far we showed that the quantum decay rates are bounded by the asymptotic extrema of the time-averaged damping. The following questions were raised in [Sjo00, AschLeb, Anan10] concerning their semiclassical distribution.

(1) What are the possible accumulation points of the quantum decay rates when $\hbar \rightarrow 0$ ? In particular, are there sequences of decay rates $(\operatorname{Im} z(\hbar) / \hbar)_{\hbar \rightarrow 0}$ converging to the extremal values $q_{ \pm}$?

(2) Do the quantum decay rates admit an asymptotic distribution when $\hbar \rightarrow 0$ ? Namely, for a given interval $I \subset\left[q_{-}, q_{+}\right]$and $1 \gg \epsilon(\hbar) \gg \hbar$, does the ratio

$$
\frac{\#\left\{z \in \operatorname{Spec} P(\hbar),|\operatorname{Re} z-1 / 2| \leq \epsilon, \frac{\operatorname{Im} z}{\hbar} \in I\right\}}{\#\{z \in \operatorname{Spec} P(\hbar),|\operatorname{Re} z-1 / 2| \leq \epsilon\}}
$$

have a limit when $\hbar \rightarrow 0$ ? Is this limit distribution related with the value distributions of the averages $\langle q\rangle_{t}$ ?

\section{Spectral estimates on Anosov manifolds}

The above questions are open in general. In order to get more precise informations on the spectrum of $P(\hbar)$, one needs to make specific assumptions on the geodesic flow on $X$. For instance, the case of a completely integrable dynamics has been considered by Hitrik-Sjöstrand in a sequence of papers (see e.g. HitSjo08 and reference therein). The case of nearly-integrable dynamics including KAM invariant tori has been studied by Hitrik-Sjöstrand-Vũ Ngọc [HSVN07]. In these cases, one can transform the Hamiltonian flow into a normal form near each invariant torus, which leads to a precise description of the spectrum "generated" by this torus. A Weyl law for the quantum decay rates was recently obtained in [HitSjo11] (for skew-adjoint perturbations $i \theta(\hbar) \mathrm{Op}_{\hbar}(q)$, with $\left.\theta(\hbar) \ll \hbar\right)$. On the other hand, Asch-Lebeau [AschLeb] addressed Question 1 for the case of the 2-dimensional standard sphere. They show that, if the damping function $q$ has real analytic real and imaginary parts, there is 


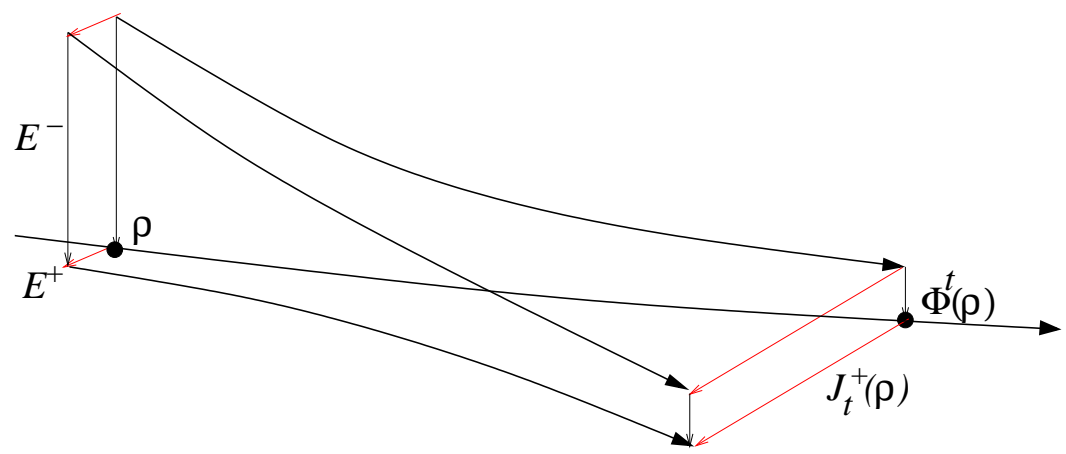

Figure 3.1. Structure of the Anosov flow near an orbit $\Phi^{t}(\rho)$

generically a spectral gap $\gamma>0$ : for $\hbar$ small enough, all eigenvalues $z_{n} \in D(1 / 2, C \hbar)$ have quantum decay rates

$$
\operatorname{Im} z_{n} / \hbar \in\left[q_{-}+\gamma, q_{+}-\gamma\right]
$$

The proof proceeds by applying a complex canonical transformation, such that the real part of the pulled-back damping function takes values in the above interval. In this case, the range of the quantum decay rates is strictly smaller than the range of classical decay rates.

We will see below that such a spectral gap may also occur in the case of Anosov geodesic flows, thanks to a different mechanism, namely a hyperbolic dispersion property due to the instability of the classical flow (see Thms 15] and 18 below).

In the next section we recall the definition and properties of Anosov manifolds.

3.1. A short reminder on Anosov manifolds [KatHas95]. At the "antipode" of the completely integrable case, one finds the "strongly chaotic" flows, namely the Anosov (or uniformly hyperbolic) flows. Uniform hyperbolicity means that at each point $\rho \in S^{*} X$ there exists a splitting of the tangent space,

$$
T_{\rho} S^{*} X=\mathbb{R} H_{p}(\rho) \oplus E^{+}(\rho) \oplus E^{-}(\rho),
$$

where $H_{p}(\rho)$ is the Hamiltonian vector field, $E^{ \pm}(\rho)$ are the unstable and stable subspaces at the point $\rho$; they both have dimension $d-1$, and are uniformly transverse to e.o.. The families $\left\{E^{ \pm}(\rho), \rho \in S^{*} X\right\}$ form the unstable/stable distributions, they are invariant w.r.to the flow, Hölder continuous, and are characterized by the following property: there exists $C, \lambda>0$ such that

$$
\forall \rho \in S^{*} X, \forall v \in E^{\mp}(\rho), \forall t>0, \quad\left\|d \Phi_{\rho}^{ \pm t} v\right\| \leq C e^{-\lambda t}\|v\| .
$$

As was shown by Hadamard, such a geodesic flow is obtained if the manifold $(X, g)$ has a negative sectional curvature. The long time properties of such a strongly chaotic flow are well understood. In particular, Hopf proved that this geodesic flow is ergodic w.r.to the Liouville measure on $S^{*} X$.

Definition 10. Call $\mu_{L}$ the normalized Liouville measure on $S^{*} X$. Then, the geodesic flow $\Phi^{t}$ on $S^{*} X$ is ergodic (w.r.to $\mu_{L}$ ) iff, for any continuous function $q$ on $S^{*} X$,

$$
\text { for } \mu_{L}-\text { almost every } \rho \in S^{*} X, \quad \lim _{t \rightarrow \infty}\langle q\rangle_{t}(\rho)=\bar{q} \stackrel{\text { def }}{=} \mu_{L}(q) \text {. }
$$


The following quantities provide quantitative measures of the hyperbolicity:

$$
\begin{aligned}
\text { the maximal expansion rate } & \lambda_{\max } \stackrel{\text { def }}{=} \lim _{t \rightarrow \infty} \sup _{\rho \in S^{*} X} \frac{1}{t} \log \left\|d \Phi_{\rho}^{t}\right\|, \\
\text { the unstable Jacobian } & J^{+}(\rho, t)=\operatorname{det}\left(d \Phi^{t} \uparrow_{E^{+}(\rho)}\right), \quad t>0, \\
\text { and its infinitesimal version } & \varphi^{+}(\rho) \stackrel{\text { def }}{=} \frac{d}{d t} \log J^{+}(\rho, t) \uparrow_{t=0}, \\
\text { the minimal expansion rate } & \nu_{\min } \stackrel{\text { def }}{=} \frac{1}{d-1} \lim _{t \rightarrow \infty} \min _{\rho \in S^{*} X} \frac{1}{t} \log J^{+}(\rho, t) .
\end{aligned}
$$

The Jacobian depends on the choice of norms on the spaces $T_{\rho} S^{*} X$. However, its long time asymptotics is independent of this choice. In variable curvature these rates are related by $\lambda_{\max } \geq \nu_{\min } \geq \lambda>0$. In particular, if the positive Lyapunov exponents are not all equal, one has $\nu_{\min }<\lambda_{\max }$.

A particular class of Anosov manifolds consists in quotients of the $d$-dimensional hyperbolic space $\mathbb{H}^{d}$ by co-compact subgroups of its isometry group. These manifolds have a constant curvature $-\Lambda^{2}$. Using the natural norms on $T_{\rho} S^{*} X$, one then has

$$
J^{+}(\rho, t)=e^{t(d-1) \Lambda}, \quad \varphi^{+}(\rho)=\Lambda(d-1), \quad \lambda_{\max }=\nu_{\min }=\Lambda .
$$

In this case the contraction/expansion are both homogeneous (independent of the point $\rho$ ) and isotropic (independent in the direction).

Let us finally notice that the study of the operator $P(\hbar)$ on an Anosov manifold belongs to the field of "quantum chaos". The methods we will use below occur in various problems of this field, e.g. the study of eigenstates of the Laplacian $P_{0}(\hbar)$ on such manifolds [Zel09].

3.2. Fractal Weyl upper bounds for the quantum decay rates. In this section we address Question 2, that is the asymptotic distribution of the quantum decay rates, for the case of Anosov manifolds.

3.2.1. Typical quantum decay rates for ergodic flows. A basic property of the geodesic flow on any Anosov manifold is ergodicity. Sjöstrand has studied the asymptotic disribution of the quantum decay rates for any ergodic geodesic flow [Sjo00], and obtained the following result.

Theorem 11. Sjo00] Assume the geodesic flow on $S^{*} X$ is ergodic w.r.to the Liouville measure. Then, For any $C>0, \epsilon>0$, one has as $\hbar \rightarrow 0$,

$$
\#\left\{z \in \operatorname{Spec} P(\hbar) \cap D(1 / 2, C \hbar), \frac{\operatorname{Im} z}{\hbar} \notin[\bar{q}-\epsilon, \bar{q}+\epsilon]\right\}=o\left(\hbar^{-d+1}\right) .
$$

Comparing this bound with the Weyl law (2.1) shows that, in the semiclassical limit, almost all the quantum decay rates are close to the phase space average $\bar{q}$. The latter could be called the typical value for quantum decay rates, while values at finite distance from $\bar{q}$ are atypical. The asymptotic distribution is simply the delta measure $\delta_{\bar{q}}$.

Remark 12. As was noticed in Anan10, this concentration result could be seen as a "nonperturbative version" of quantum ergodicity. The latter property states

\footnotetext{
${ }^{4}$ Usually one normalizes the metrics on $\mathbb{H}^{d}$ so that the curvature is -1 . We prefer to keep track of the curvature in our notations.
} 
that almost all the eigenstates $u_{n}^{0}$ of $P_{0}(\hbar)$ with eigenvalues $z_{n}^{0} \in D(1 / 2, C \hbar)$ satisfy $\left\langle u_{n}^{0}, \mathrm{Op}_{\hbar}(q) u_{n}^{0}\right\rangle=\bar{q}+o_{\hbar \rightarrow 0}(1)$. A naive perturbation theory argument would predict that, when switching on $i \hbar \mathrm{Op}_{\hbar}(q)$, the eigenvalues $z_{n}^{0}$ move to $z_{n}=z_{n}^{0}+$ $i \hbar\left\langle u_{n}^{0}, \mathrm{Op}_{\hbar}(q) u_{n}^{0}\right\rangle+\mathcal{O}\left(\hbar^{2}\right)$, so that the quantum decay rates $\operatorname{Im} z_{n} / \hbar=\left\langle u_{n}^{0}, \mathrm{Op}_{\hbar}(q) u_{n}^{0}\right\rangle+$ $\mathcal{O}(\hbar)$, the RHS being equal to $\bar{q}+o(1)$ for almost all $n$. Of course, this argument does not apply because the perturbation $i \hbar \mathrm{Op}_{\hbar}(q)$ is much stronger than the mean spacing between successive eigenvalues.

To prove the above theorem one relates the counting of quantum decay rates with the value distribution of the quantum averages $\langle q\rangle_{t}$ on $S^{*} X$, that is the volumes

$$
\mu_{L}\left\{\rho \in S^{*} X,\langle q\rangle_{t}(\rho) \geq \alpha\right\}, \quad \alpha \in \mathbb{R}, t>0 .
$$

Indeed, the main intermediate result in the proof is the bound

$\#\left\{z \in \operatorname{Spec} P(\hbar) \cap D(1 / 2, C \hbar), \frac{\operatorname{Im} z}{\hbar} \geq \alpha\right\} \leq C_{t, \delta} \hbar^{-d+1} \mu_{L}\left\{\rho \in S^{*} X,\langle q\rangle_{t}(\rho) \geq \alpha-\delta\right\}$,

which holds for any fixed $t>0$ and $\delta>0$ (this bound holds independently of the ergodicity assumption). Ergodicity then implies that the value distribution of $\langle q\rangle_{t}$ converges to $\delta_{\bar{q}}$ when $t \rightarrow \infty$; in particular, for any $\alpha>\bar{q}$ the volume (3.5) decays to zero when $t \rightarrow \infty$. We have then obtained the bound $o\left(\hbar^{-d+1}\right)$ for the quantum decay rates $\geq \alpha>\bar{q}$. The case of values $\leq \alpha<\bar{q}$ is treated analogously.

3.2.2. Large deviation estimates for Anosov flows. For an Anosov flow, one has more precise estimates for the volumes (3.5), which can then induce sharper bounds on the number of the atypical quantum decay rates. These volume estimate take the form of large deviation estimates. Let us introduce some notations. We call $\mathcal{M}$ the set of $\Phi^{t}$-invariant probability measures on $S^{*} X$. For each such measure $\mu$, we denote by $h_{K S}(\mu)$ its Kolmogorov-Sinai entropy: this is a nonnegative number, which measures the complexity of a $\mu$-typical trajectory [KatHas95]. Then, we define the rate function $\tilde{H}: \mathbb{R} \rightarrow \mathbb{R}$ as follows:

$$
\forall s \in \mathbb{R}, \quad \tilde{H}(s) \stackrel{\text { def }}{=} \sup \left\{h_{K S}(\mu)-\mu\left(\varphi^{+}\right), \mu \in \mathcal{M}, \mu(q)=s\right\},
$$

where we recall that $\varphi^{+}$is the infinitesimal unstable Jacobian (3.3). We are now ready to state our large deviation result.

Theorem 13. Kif90 Assume the geodesic flow on $S^{*} X$ is Anosov. Then, for any closed interval $I \subset \mathbb{R}$ and for any $q \in C^{\infty}\left(S^{*} X\right)$, the time averages $\langle q\rangle_{t}$ satisfy

$$
\limsup _{t \rightarrow \infty} \frac{1}{t} \log \mu_{L}\left\{\rho \in S^{*} X,\langle q\rangle_{t}(\rho) \in I\right\} \leq \sup _{s \in I} \tilde{H}(s) .
$$

The rate function $\tilde{H}$ is continuous on $\left[q_{-}, q_{+}\right]$, smooth and strictly concave on $\left(q_{-}, q_{+}\right)$, negative except at the point $\tilde{H}(\bar{q})=0$, satisfies $\tilde{H}(s) \geq-\sup _{\mu \in \mathcal{M}} \mu\left(\varphi^{+}\right)$ for $s \in\left[q_{-}, q_{+}\right]$, and is equal to $-\infty$ outside $\left[q_{-}, q_{+}\right]$. As a consequence of (3.8), for any $\alpha \geq \bar{q}$ and $\epsilon>0$ arbitrary small, there exists $T_{\alpha, \epsilon}>0$ such that

$$
\forall t \geq T_{\alpha, \epsilon}, \quad \mu_{L}\left\{\rho \in S^{*} X,\langle q\rangle_{t}(\rho) \geq \alpha\right\} \leq e^{t(\tilde{H}(\alpha)+\epsilon)} .
$$

Due to the negativity of $\tilde{H}(\alpha)$ for $\alpha \neq \bar{q}$, we see that the probability of $\langle q\rangle_{t}$ taking atypical values decays exponentially when $t \rightarrow \infty$. 
3.2.3. Fractal Weyl upper bounds on Anosov manifolds. Using these large deviation estimates, Anantharaman Anan10 improved Thm 11 by letting the averaging time grow with $\hbar$ in a controlled way. The optimal time is the Ehrenfest time

$$
T=T_{E h r}=(1-2 \epsilon) \frac{\log 1 / \hbar}{\lambda_{\max }},
$$

where $\lambda_{\max }$ is the largest expansion rate (3.2) and $\epsilon>0$ arbitrary small. What is the signification of this time? For any $f \in S^{-\infty}\left(T^{*} X\right)$ supported in an $\epsilon$-neighbourhood of $S^{*} X$, the classically evolved observable $f \circ \Phi^{t}$ remains in the "good" symbol class 5 $S_{1 / 2-\epsilon}^{-\infty}\left(T^{*} X\right)$ uniformly for times $|t| \leq T_{E h r} / 2$. In turn, the symmetric averages

$$
\langle f\rangle_{t, s y m}=\frac{1}{t} \int_{-t / 2}^{t / 2} f \circ \Phi^{s} d s
$$

belong to $S_{1 / 2-\epsilon}^{-\infty}\left(T^{*} X\right)$ for $|t| \leq T_{E h r}$, and this time is sharp.

Let us insist on the fact that controlling the time evolution up to times $t \asymp$ $\log 1 / \hbar$ is a crucial ingredient in order to obtain refined spectral estimates on Anosov manifolds. This will also be the case in Sections 3.3 and 3.4 when proving hyperbolic dispersion estimates and spectral gaps.

The pseudodifferential calculus on $\Psi_{1 / 2-\epsilon}^{2}(X)$ allows to extend the validity of the bound (3.6) up to the Ehrenfest time. The large deviation estimate (3.9) then leads to the following fractal Weyl upper bound for the number of atypical quantum decay rates.

Theorem 14. Anan10] Assume the geodesic flow on $S^{*} X$ is Anosov. Then, for any $\alpha \geq \bar{q}$ and $\epsilon>0$, one has for $\hbar$ small enough

$$
\#\left\{z \in \operatorname{Spec} P(\hbar) \cap D(1 / 2, C \hbar), \frac{\operatorname{Im} z}{\hbar} \geq \alpha\right\} \leq \hbar^{-\frac{\tilde{H}(\alpha)}{\lambda_{\max }}+(1-d)-\epsilon},
$$

where $\tilde{H}$ is the rate function (3.7). A similar expression holds when counting quantum decay rates smaller than $\alpha^{\prime} \leq \bar{q}$.

Since $-\tilde{H}(\alpha)>0$ for all $\alpha>\bar{q}$, this upper bound improves the bound $o\left(\hbar^{-d+1}\right)$ of Thm. 11 by a fractional power of $\hbar$ (the name "fractal Weyl upper bound" takes its origin in the counting of resonances of chaotic scattering systems [SjoZwo07]). For any $\alpha \in\left[\bar{q}, q_{+}\right]$one has $\tilde{H}(\alpha) \geq-\sup _{\mu} \mu\left(\varphi^{+}\right) \geq-\lambda_{\max }(d-1)$, so the exponent of $\hbar$ in (3.12) is negative, allowing the presence of (many) quantum decay rates arbitrary close to $q_{+}$.

These fractal upper bounds are not expected to be sharp in general [Anan10. In the next sections we will be able, under certain conditions, to exclude the possibility of quantum decay rates near $q_{+}$(or near $q_{-}$).

\footnotetext{
${ }^{5}$ For any $k \in \mathbb{R}, \delta \in[0,1 / 2)$, the symbol class $S_{\delta}^{k}\left(T^{*} X\right)$ consists of functions $g(x, \xi ; \hbar)$ which may become more and more singular when $\hbar \rightarrow 0$, but in a controlled way:

$$
\forall \alpha, \beta \in \mathbb{N}^{d}, \forall \rho \in T^{*} X, \quad\left|\partial_{x}^{\alpha} \partial_{\xi}^{\beta} g(\rho)\right| \leq C_{\alpha} \hbar^{-\delta(|\alpha|+|\beta|)}(1+|\xi|)^{k-|\beta|} .
$$

In this class one can still use pseudodifferential calculus, and the expansions in powers of $\hbar$ make sense [EvZw, Sec. 4.3].
} 
3.3. A pressure criterium for a spectral gap. The result of Thm 9, namely the fact that all quantum decay rates belong to the interval $\left[q_{-}-o(1), q_{+}+o(1)\right]$, was obtained by studying the norm of the propagator $V^{t}=e^{-i t P / \hbar}$ for times $|t| \gg 1$ independent of $\hbar$. Indeed, the sequence of equalities (2.5) directly leads to the upper bound

$$
\operatorname{Im} z_{n} / \hbar \leq q_{+}+\epsilon .
$$

The crucial semiclassical ingredient in (2.5) is the second equality connecting the $L^{2}$ norm of the PDO $\tilde{B}(t) \chi\left(P_{0}\right)$ with the supremum of its (principal) symbol. Hence, one cannot hope to improve the bound (3.13) as long as $\tilde{B}(t) \chi\left(P_{0}\right)$ remains a "good" PDO, which is the case if the time $t \leq T_{E h r} / 2$. To improve on (3.13) we will investigate the propagator $V^{t}$ for "large logarithmic times", namely

$$
t \sim \mathcal{K} \log 1 / \hbar, \quad \text { with } \mathcal{K}>0 \text { large (but independent of } \hbar \text { ) }
$$

For such times, the function $\tilde{b}(t) \chi\left(p_{0}\right)$ oscillates on scales much smaller than $\hbar$, so it cannot belong to any decent symbol class. As a result, the norm of $\tilde{B}(t) \chi\left(P_{0}\right)$ is a priori unrelated with that function. However, using the hyperbolicity of the flow one can prove an upper bound for the propagator $V^{t}$ in terms of a certain topological pressure depending on the damping and the hyperbolicity, and then obtain a constraint on the quantum decay rates.

Before stating our bound, let us first introduce the notion of topological pressure associated with the flow $\Phi^{t}$ on $S^{*} X$. For any observable $f \in C\left(S^{*} X\right)$, the pressure $\mathcal{P}(f)=\mathcal{P}\left(f, \Phi^{t} \Gamma_{S^{*} X}\right)$ can be defined by

$$
\mathcal{P}(f) \stackrel{\text { def }}{=} \sup \left\{h_{K S}(\mu)+\mu(f), \mu \in \mathcal{M}\right\} .
$$

Notice that the definition (3.7) of the rate function $\tilde{H}(s)$ is very similar. The pressure is also given by the growth rate of weighted sums over long closed geodesics:

$$
\mathcal{P}(f)=\lim _{T \rightarrow \infty} \frac{1}{T} \log \sum_{T \leq|\gamma| \leq T+1} e^{\int_{\gamma} f} .
$$

The pressure appearing in the next theorem is associated with the function $f=$ $q-\varphi^{+} / 2$, where $\varphi^{+}$is the infinitesimal unstable Jacobian (3.3), it thus mixes the damping and hyperbolicity.

Theorem 15. Sche10] Let $X$ be an Anosov manifold, and $q \in S^{0}\left(T^{*} X\right)$ a damping function.

Assume the following (purely classical) inequality holds:

$$
\mathcal{P}\left(q-\varphi^{+} / 2\right)<q_{+} .
$$

Then, for any $\epsilon>0$, there exists $N>0$ such that, for $\hbar$ small enough, the following resolvent bounds:

$$
\forall z \in D(1 / 2, C \hbar) \cap\left\{\operatorname{Im} z / \hbar \geq \mathcal{P}\left(q-\varphi^{+} / 2\right)+\epsilon\right\}, \quad\left\|(P(\hbar)-z)^{-1}\right\| \leq \hbar^{-N} .
$$

\footnotetext{
${ }^{6}$ The sum over long closed geodesics is analogous to a partition function in statistical mechanics, with the time $T$ corresponding to the volume of the system. This explains why the growth rate is called a "pressure".
} 


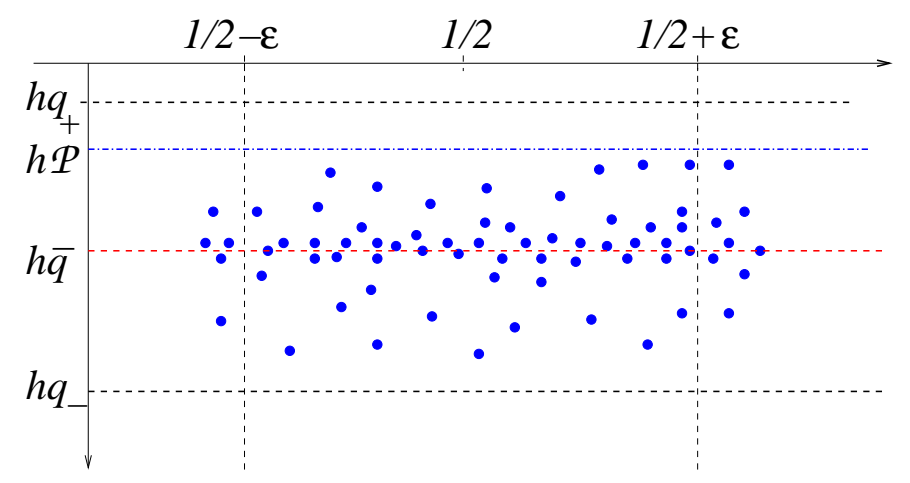

Figure 3.2. Spectral gap in case the pressure $\mathcal{P}\left(q-\varphi^{+} / 2\right)<q_{+}$.

As a consequence, all quantum decay rates for $z_{n}(\hbar) \in D(1 / 2, C \hbar)$ satisfy

$$
\frac{\operatorname{Im} z_{n}(\hbar)}{\hbar} \leq \mathcal{P}\left(q-\varphi^{+} / 2\right)+\epsilon
$$

in particular we have a spectral gap.

In the next subsection we present situations for which the inequality (3.16) is satisfied. The proof of this theorem was inspired by a similar result in the case of resonances in chaotic scattering [NZ2, NZ3]. We give some hints of the proof in $\$ 3.3 .2$.

Let us now apply this bound to the damped wave equation, that is take $q(x, \xi)=$ $-a(x)$.

Corollary 16. SSche10] Consider the DWE on $X$ with nontrivial damping $a \geq 0$. Assume that the GCC fails $\left(a_{-}=0\right)$, but that the condition $\mathcal{P}\left(-a-\varphi^{+} / 2\right)<0$ holds true.

Then the DWE has a spectral gap, and the energy decays exponentially for regular initial data, as in (1.10).

3.3.1. Conditions for a spectral gap. The inequality (3.16) can hold only if the damping function $q$ varies "sufficiently" around its average value $\bar{q}$. Indeed, one always has $\mathcal{P}\left(-\varphi^{+} / 2\right)>0$ for an Anosov flow, so by adding a constant dampint $q \equiv \bar{q}$ one gets

$$
\mathcal{P}\left(\bar{q}-\varphi^{+} / 2\right)=\bar{q}+\mathcal{P}\left(-\varphi^{+} / 2\right)>\bar{q}=q_{+} .
$$

The pressure depends continuously of $q$ (in the $C^{0}$ topology), so if $q=\bar{q}+\delta q$ with $\|\delta q\|_{C^{0}}$ small, the inequality (3.16) will not be satisfied.

Let us consider the case of the DWE as in Corollary 16. The failure of the GCC is equivalent to the fact that the set of undamped trajectories $K(1.9)$ is nonempty. This set is flow-invariant and closed, so one can define the pressure $\mathcal{P}\left(-\varphi^{+} / 2, \Phi^{t} \uparrow_{K}\right)$ associated with the flow $\Phi^{t}$ restricted on $K$. It was shown in Sche11 that if we multiply the damping $a(x)$ by a large constant $C>0$ (this does not modify the set $K)$, then

$$
\lim _{C \rightarrow \infty} \mathcal{P}\left(-C a-\varphi^{+} / 2\right)=\mathcal{P}\left(-\varphi^{+} / 2, \Phi^{t} \uparrow_{K}\right) .
$$

If the set $K$ is thin enough, the pressure $\mathcal{P}\left(-\varphi^{+} / 2, \Phi^{t} \uparrow_{K}\right)$ is negative (for instance, if $K$ consists in a single closed geodesic, the pressure is equal to the average of $-\varphi^{+} / 2$ along the geodesic, which is negative). In that case, the above limit shows that the pressure $\mathcal{P}\left(-C a-\varphi^{+} / 2\right)$ is also negative if $C \gg 1$. 
3.3.2. Proof of the pressure bound: decomposing into "symbolic paths". In this section we explain the strategy of proof of Thm 15. As mentioned before, we want to bound the norm of the propagator for large logarithmic times. We will prove the following

Lemma 17. Choose $\epsilon>0$. Then, for an energy cutoff $\chi_{0}$ of small enough support, and a large enough constant $\mathcal{K}>0$, for $\hbar<\hbar_{\epsilon}$ one has the bound

$$
\left\|V^{t} \chi_{0}\left(P_{0}\right)\right\| \leq e^{t\left(\mathcal{P}\left(q-\varphi^{+} / 2\right)+\epsilon\right)}, \quad t \sim \mathcal{K} \log 1 / \hbar .
$$

From there, obtaining the resolvent estimate (3.17) is relatively straightforward.

Proof. Our aim is to bound the norm $\left\|V^{t} u_{0}\right\|$ for a normalized state $u_{0}$ microlocalized in $p_{0}^{-1}([1 / 2 \pm \delta])$, and a time $t \sim \mathcal{K} \log 1 / \hbar$. We may assume that $u_{0}$ is microlocalized in an open subset $W$ of diameter $\leq \delta$. We will first decompose $u_{0}$ in an adapted basis of local "momentum" modes $\left\{e_{\eta}, \eta \in \mathbb{R}^{d},|\eta| \leq \delta\right\}$. Here the parameter $\eta$ is the "momentum coordinate" in a Darboux coordinate frame $\{(y, \eta),|y| \leq \delta,|\eta| \leq \delta\}$ in $W$, chosen such that $\eta_{1}=p_{0}-1 / 2$ represents the energy, $y_{1}$ the time, and the "momentum" Lagrangian leaves $\Lambda_{\eta_{0}}=\left\{\left(y, \eta_{0}\right)\right\}$ are close the unstable foliation. The momentum states $e_{\eta}$ are Lagrangian states supported by the leaves $\Lambda_{\eta}$, of norms $\mathcal{O}(1)$. The decomposition of $u_{0}$ in this "basis" reads

$$
u_{0}=\int_{|\eta| \leq \delta} \hat{u}_{0}(\eta) e_{\eta} \frac{d \eta}{(2 \pi \hbar)^{d / 2}}+\mathcal{O}\left(\hbar^{\infty}\right),
$$

where the factor $(2 \pi \hbar)^{-d / 2}$ ensures that $\left\|\hat{u}_{0}\right\|_{L^{1}}=\mathcal{O}(1)$. The reason for this decomposition is that we are able to precisely control the evolution of each individual state $e_{\eta}$, up to times $t \sim \mathcal{K} \log 1 / \hbar$. The state $V^{t} e_{\eta}$ is a Lagrangian state supported on the Lagrangian leaf $\Phi^{t}\left(\Lambda_{\eta}\right)$. Due to the hyperbolicity of the flow, when $t$ grows the leaf $\Phi^{t}\left(\Lambda_{\eta}\right)$ expands exponentially fast along the unstable directions, and converges to the unstable foliation. To analyze the state $V^{t} e_{\eta}$, we can view as the combination of many local Lagrangian states associated with bounded pieces of $\Phi^{t}\left(\Lambda_{\eta}\right)$. A convenient bookkeeping consists in using a partition of unity in $p_{0}^{-1}([1 / 2 \pm \delta])$. One first covers this region by a finite family of open subsets $\left\{W_{j}, j=1, \ldots, J\right\}$ of diameters $\leq \delta$, and then constructs a smooth partition of unity $\left\{\pi_{j} \in C_{c}^{\infty}\left(W_{j},[0,1]\right), j=1, \ldots, J\right\}$ adapted to this cover: $\sum_{j=1}^{J} \pi_{j}=1$ near $p_{0}^{-1}([1 / 2 \pm \delta])$. This partition is quantized into $\left\{\Pi_{j} \stackrel{\text { def }}{=} \mathrm{Op}_{\hbar}\left(\pi_{j}\right), j=1, \ldots, J\right\}$, such that, for any energy cutoff $\chi$ any supported in $[1 / 2 \pm \delta]$,

$$
\sum_{j=1}^{J} \Pi_{j} \chi\left(P_{0}\right)=\chi\left(P_{0}\right)+\mathcal{O}_{L^{2} \rightarrow L^{2}}\left(\hbar^{\infty}\right) .
$$

We use this quantum partition to split the propagator $V^{n}$ into "symbolic paths":

$$
V^{n} e_{\eta}=\sum_{\alpha_{1}, \ldots, \alpha_{n}=1}^{J} V_{\alpha_{1} \cdots \alpha_{n}} e_{\eta}+\mathcal{O}\left(\hbar^{\infty}\right), \quad V_{\alpha_{1} \cdots \alpha_{n}}=\Pi_{\alpha_{n}} V \Pi_{\alpha_{n-1}} \cdots V \Pi_{\alpha_{1}} V .
$$

For each symbolic sequence $\boldsymbol{\alpha}=\alpha_{1} \cdots \alpha_{n}$, the state $V_{\boldsymbol{\alpha}} e_{\eta}$ is a Lagrangian state supported on the Lagrangian leaf $\Lambda_{\eta}^{\alpha}$ obtained from $\Lambda_{\eta}$ by a sequence of $n$ evolutions by $\Phi^{1}$ and truncations on $W_{\alpha_{k}}$. The full evolved leaf is given by $\Phi^{t}\left(\Lambda_{\eta}\right)=\bigcup_{|\boldsymbol{\alpha}|=n} \Lambda_{\eta}^{\alpha}$. If $\Lambda_{\eta}^{\boldsymbol{\alpha}}$ is empty $V_{\boldsymbol{\alpha}} e_{\eta}=\mathcal{O}\left(\hbar^{\infty}\right)$. Otherwise ( $\boldsymbol{\alpha}$ "admissible"), $\Lambda_{\eta}^{\boldsymbol{\alpha}}$ is a leaf of diameter $\leq \delta$, close to the unstable foliation. One can compute the amplitude of 
the Lagrangian state $V_{\boldsymbol{\alpha}} e_{\eta}$ to any order in $\hbar$. It is governed by both the accumulated damping and accumulated instability along the "path" $\boldsymbol{\alpha}$, leading to the norm estimate

$$
\begin{gathered}
\left\|V_{\boldsymbol{\alpha}} e_{\eta}\right\| \leq C b(\boldsymbol{\alpha}) J^{+}(\boldsymbol{\alpha})^{-1 / 2}, \quad \text { where } \\
b(\boldsymbol{\alpha})=\prod_{k=1}^{n} b\left(\alpha_{k}\right), \quad b(j)=\max _{\rho \in W_{j}} b(1, \rho), \quad \text { and similarly for }\left(J^{+}\right)^{-1 / 2} .
\end{gathered}
$$

Applying the triangular inequality to (3.22), we obtain a bound on the norm of $V^{n} e_{\eta}$ which can be related with the topological pressure:

$$
\left\|V^{n} e_{\eta}\right\| \leq C \sum_{\boldsymbol{\alpha} \text { admis. }}^{J} b(\boldsymbol{\alpha}) J^{+}(\boldsymbol{\alpha})^{-1 / 2} \leq \exp \left\{n\left(\mathcal{P}\left(q-\varphi^{+} / 2\right)+\mathcal{O}(\delta)\right)\right\} .
$$

Inserting this bound in the expansion (3.20) we get the same bound for $V^{n} u_{0}$ with an extra factor $\hbar^{-d / 2}$; however, this factor is smaller than $e^{n \delta}$ if we take $n \sim \mathcal{K} \log 1 / \hbar$ and $\mathcal{K}$ large enough, in which case the bound (3.25) also applies to $\left\|V^{n} u_{0}\right\|$. This ends the proof of the norm bound (3.19).

Had we inserted (3.23) in the expansion (3.20) before summing over $\boldsymbol{\alpha}$, we would have obtained the following hyperbolic dispersion estimate:

$$
\left\|V_{\boldsymbol{\alpha}}\right\| \leq \min \left(b(\boldsymbol{\alpha}), C \hbar^{-d / 2} b(\boldsymbol{\alpha}) J^{+}(\boldsymbol{\alpha})^{-1 / 2}\right), \quad|\boldsymbol{\alpha}| \leq \mathcal{K} \log 1 / \hbar .
$$

This type of estimate was first proved in the case of the undamped operator $P_{0}(\hbar)$ on an Anosov manifold [Anan08, AN1]. The adaptation to the case of the damped wave equation was written in [Sche10]. A similar estimate was also shown in [NZ2] in the case of chaotic scattering.

3.4. A "thickness" condition for a spectral gap. In this section we present a spectral gap condition expressed only in terms of the set of undamped trajectories (1.9), but independently of the strength of the variations of the damping (as opposed to the "pressure condition" (3.16)). A proof of this result will appear in a forthcoming publication.

To state our result in the context of a damping function $q(x, \xi)$, we introduce the set of "least damped trajectories"

$$
K=\overline{\bigcup\left\{\operatorname{supp} \mu, \mu \in \mathcal{M}, \mu(q)=q_{+}\right\}},
$$

which generalizes 7 the set (1.9). Our condition for a spectral gap depends on a topological pressure associated with the flow $\Phi^{t} \uparrow_{K}$. We use quantities defined in $\$ 3.1$.

Theorem 18. Let $X$ be an Anosov manifold, and $q \in S^{0}\left(T^{*} X\right)$ a damping function. Assume the following condition holds:

$$
\mathcal{P}\left(-\varphi^{+}, \Phi^{t} \uparrow_{K}\right)<(d-1)\left(\frac{\nu_{\min }}{2}-\lambda_{\max }\right) .
$$

Then, there exists $\gamma>0$ ("the gap"), $C>0$ and $N>0$ such that, for $\hbar>0$ small enough, the following resolvent bound holds:

$$
\forall z \in D(1 / 2, C \hbar) \cap\left\{\operatorname{Im} z / \hbar \geq q_{+}-\gamma\right\}, \quad\left\|(P(\hbar)-z)^{-1}\right\| \leq C \hbar^{-N} .
$$

\footnotetext{
${ }^{7}$ The two definitions are not strictly equal [Sche11], but this subtlety will be irrelevant here.
} 
As a consequence, all eigenvalues of $P(\hbar)$ in $D(1 / 2, C \hbar)$ satisfy

$$
\operatorname{Im} z_{n} / \hbar \leq q_{+}-\gamma
$$

so we have a spectral gap.

In the framework of the damped wave equation with (nontrivial) damping function $a(x) \geq 0$, this gives the following

Corollary 19. Assume that the set of undamped trajectories (1.9) is such that the condition (3.27) holds. Then, there exists $\gamma>0, C>0$ and $N>0$ such that

$$
\left\|(\tau-\mathcal{A})^{-1}\right\|_{L^{2} \rightarrow L^{2}} \leq C \tau^{N}, \quad \forall \tau \in\{|\operatorname{Re} \tau| \geq C, \operatorname{Im} \tau \geq-\gamma\} .
$$

As a consequence, the energy decays exponentially for regular initial data, as in (1.10).

On a manifold of constant negative curvature $-\Lambda^{2}$, the condition (3.27) takes the form

$$
h_{\text {top }}\left(\Phi^{t} \uparrow_{K}\right)<(d-1) \Lambda / 2
$$

where $h_{\text {top }}$ is the topological entropy of the flow on $K$. It is usually interpreted as a measure of the "complexity" of the flow on $K$; we rather see it as a measure of the "thickness" of $K$. This bound reminds us of the lower bound obtained by Anantharaman when describing the localization of eigenstates of the Laplacian on Anosov manifolds [Anan08]: in constant curvature, she shows the semiclassical measures associated with any sequence of eigenstates cannot be supported on a set of entropy smaller than $(d-1) \Lambda / 2$, thus forbidding the eigenstates from being too localized. Following the thread of Remark 12, the spectral gap condition (3.29) could be seen as a "nonperturbative analogue" of this delocalization result.

Remark 20. For certain Anosov manifolds of dimension $d \geq 3$, the condition (3.27) can never be satisfied. Indeed, the variational formula (3.15) shows that, for any closed invariant set $K^{\prime} \subset S^{*} X$, the pressure

$$
\mathcal{P}\left(-\varphi^{+}, \Phi^{t}\left\lceil_{K^{\prime}}\right) \geq-\sup _{\mu \in \mathcal{M}} \mu\left(\varphi^{+}\right) \geq-(d-1) \lambda_{\max } .\right.
$$

If the instability along $E^{+}$is very anistropic (meaning that the largest and smallest positive Lyapunov exponents are very different), then $(d-1) \lambda_{\max }-\sup _{\mu \in \mathcal{M}} \mu\left(\varphi^{+}\right)$ can be strictly larger than $\frac{(d-1) \nu_{\min }}{2}$, in which case

$$
\mathcal{P}\left(-\varphi^{+}, \Phi^{t} \uparrow_{K^{\prime}}\right)>(d-1)\left(\frac{\nu_{\min }}{2}-\lambda_{\max }\right) \quad \text { for any closed invariant set } K^{\prime} .
$$

This remark hints at the fact that the condition (3.27) is probably not sharp (even in 2 dimensions), except maybe on manifolds of constant curvature. Rivière has recently improved Anantharaman's lower bound on the support of semiclassical measures in variable curvature [Riv11]: he shows that any such support $S$ must satisfy $\mathcal{P}\left(-\varphi^{+} / 2, \Phi^{t} \uparrow_{S}\right) \geq 0$. If we follow the above analogy (and also following the results of 93.3 .1$)$, it seems natural to expect the following

Conjecture 21. If the set $K$ of least damped trajectories satisfies

$$
\mathcal{P}\left(-\varphi^{+} / 2, \Phi^{t} \uparrow_{K}\right)<0,
$$

then there is a spectral gap in the spectrum of $P(\hbar)$, in the sense of Thm 18 . 
In constant curvature the condition (3.30) is equivalent with (3.29). In variable curvature, it is weaker than (3.27). A Proof of (3.30) should make use of local expansion rates, instead of the globally defined rates $\lambda_{\max }$ and $\nu_{\min }$, like in Rivière's work on 2-dimensional Anosov manifolds Riv10.

In the next subsection we sketch the proof of Thm 18 .

3.4.1. Sketch of proof for Thm 18. As was the case for Thm 15, the proof proceeds by bounding the norm of the propagator $V^{t} \chi_{0}\left(P_{0}\right)$ for some logarithmic time (now the double of the Ehrenfest time, see (3.38)). In the course of the proof we will also need to control the evolution of a certain type of "microscopic" Lagrangian states. The novelty compared to the proof of Thm 15 is that we will now distinguish between two types of phase space points, the "weakly damped" vs. "strongly damped" points.

Without loss of generality 8 , we may assume that the damping function $q(x, \xi)$ is compactly supported inside $p_{0}^{-1}([1 / 2 \pm \epsilon])$. From now on we denote the Ehrenfest time by $T \stackrel{\text { def }}{=} T_{E h r}$. We fix some level $\alpha \in\left(\bar{q}, q_{+}\right)$, and consider the set of "weakly damped points"

$$
\Omega_{+, \alpha} \stackrel{\text { def }}{=}\left\{\rho \in T^{*} X,\langle q\rangle_{T, \text { sym }}(q) \geq \alpha\right\}
$$

(remember that $\langle q\rangle_{t, \text { sym }}$ is the symmetric time average (3.11)). The large deviation estimate (3.9) provides a bound on the volume of $\Omega_{+, \alpha} \cap S^{*} X$ :

$$
\mu_{L}\left(\Omega_{+, \alpha} \cap S^{*} X\right) \leq \hbar^{-\frac{\tilde{H}(\alpha)}{\lambda_{\max }}-\mathcal{O}(\epsilon)} .
$$

From the definition (3.7), $\tilde{H}\left(q_{+}\right)$is equal to the pressure $\mathcal{P}\left(-\varphi^{+}, \Phi^{t} \uparrow_{K}\right)$ appearing in (3.27). If we assume (3.27), then, by continuity of $\tilde{H}(s)$ on $\left[q_{-}, q_{+}\right]$, we may choose $\alpha \in\left(\bar{q}, q_{+}\right)$large enough such that

$$
\beta(\alpha) \stackrel{\text { def }}{=}(d-1)\left(\frac{\nu_{\text {min }}}{2}-\lambda_{\max }\right)-\tilde{H}(\alpha)>0 .
$$

We will now associate a quantum projector to the set $\Omega_{+, \alpha}$. We first symmetrize the factorization (2.3), by writing

$$
V^{t}=U^{t / 2} B_{s}(t) U^{t / 2}, \quad \text { where } \quad B_{s}(t) \quad \text { has principal symbol } b_{s}(t)=e^{t\langle q\rangle_{t, s y m}} .
$$

Although this symbol is positive, the operator $B_{s}(t)$ may not be selfadjoint; we then take its polar decomposition

$$
B_{s}(t)=W(t) A(t), \quad \text { where }\left\{\begin{array}{cl}
A(t)=\left(B_{s}(t)^{*} B_{s}(t)\right)^{1 / 2} & \text { is definite positive, } \\
W(t) & \text { is unitary. }
\end{array}\right.
$$

For the same reasons as in $\$ 3.2 .3$ (and due to the support assumption on $q$ ), the operators $B_{s}(T), A(T), W(T)$ remain "good" PDO up to the Ehrenfest time: $B_{s}(T), A(T) \in \hbar^{-C} \Psi_{1 / 2-\epsilon}^{0}(X)$, and $W(T) \in \Psi_{1 / 2-\epsilon}^{0}(X)$.

The operators $A(T)$ and $B_{s}(T)$ have the same leading symbol $b_{s}(T)=e^{T\langle q\rangle_{T, s y m}}$. Hence, to the set $\Omega_{+, \alpha}=\left\{b_{s}(T, \rho) \geq e^{\alpha T}\right\}$ we associate the spectral projector

$$
\Pi_{+}=\Pi_{+, \alpha} \stackrel{\text { def }}{=} \mathbb{1}_{A(T) \geq e^{\alpha T}}, \quad \text { and call } \Pi_{-}=I-\Pi_{+} .
$$

\footnotetext{
${ }^{8}$ Lemma 7 can be extended to logarithmic times.
} 
We use these projectors to decompose the propagator at time $2 T$ : for some energy cutoff $\chi_{0}$ we write

$$
\begin{aligned}
& V^{2 T} \chi_{0}\left(P_{0}\right)=U^{T / 2} W(T) A(T) U^{T} W(T) A(T) U^{T / 2} \chi_{0}\left(P_{0}\right) \\
& \quad=U^{T / 2} W(T) A(T)\left(\Pi_{+}+\Pi_{-}\right) U^{T} W(T)\left(\Pi_{+}+\Pi_{-}\right) A(T) U^{T / 2} \chi_{0}\left(P_{0}\right) .
\end{aligned}
$$

The RHS splits into four terms. Three terms contain at least one factor $\Pi_{-}$: for them we use the obvious bound

$$
\left\|A(T) \Pi_{-}\right\|=\left\|\Pi_{-} A(T)\right\| \leq e^{\alpha T} .
$$

The remaining term contains the factor

$$
U_{++}^{T} \stackrel{\text { def }}{=} \Pi_{+} U^{T} W(T) \Pi_{+} .
$$

The norm of this operator will be bounded by the following hyperbolic dispersion estimate, the proof of which is sketched in 3.4 .2 .

Proposition 22. Assume that for some $\alpha \in\left(\bar{q}, q_{+}\right)$the condition (3.32) holds. Fix some $\epsilon>0$. Then, if the energy cutoff $\chi_{1}$ has small enough support, for $\hbar>0$ small enough one has

$$
\left\|U_{++}^{T} \chi_{1}\left(P_{0}\right)\right\|_{L^{2} \rightarrow L^{2}} \leq \hbar^{\frac{\beta(\alpha)}{\lambda_{\max }}-\mathcal{O}(\epsilon)} .
$$

Since propagation does not modify the energy localization, if choose in (3.34) a cutoff $\chi_{0} \prec \chi_{1}$, we then have

$$
A(T) U^{T / 2} \chi_{0}\left(P_{0}\right)=\chi_{1}\left(P_{0}\right) A(T) U^{T / 2} \chi_{0}\left(P_{0}\right)+\mathcal{O}\left(\hbar^{\infty}\right) .
$$

Inserting this identity and the bounds (2.7), (3.35) and (3.36) in the identity (3.34), we get

$$
\left\|V^{2 T} \chi_{0}\left(P_{0}\right)\right\| \leq e^{2 T\left(q_{+}+\mathcal{O}(\epsilon)\right)}\left(e^{-T \beta(\alpha)}+e^{T\left(\alpha-q_{+}\right)}+e^{2 T\left(\alpha-q_{+}\right)}\right) .
$$

We may optimize this upper bound over the level $\alpha$ : the optimal value of the exponent is reached for the (unique) parameter $\alpha_{c} \in\left(\bar{q}, q_{+}\right)$solving

$$
\beta(\alpha)=q_{+}-\alpha \text {. }
$$

For any $\gamma>0$ satisfying $q_{+}-\gamma>\frac{q_{+}+\alpha_{c}}{2}$, we get (for $\hbar>0$ small enough) the following norm bound for the propagator:

$$
\left\|V^{2 T} \chi_{0}\left(P_{0}\right)\right\| \leq e^{2 T\left(q_{+}-\gamma\right)} .
$$

The proof of the resolvent estimate (3.28) is then rather straightforward.

3.4.2. Proof of the norm bound for $U_{++}^{T}$. In this last subsection we sketch the proof of Proposition 22, that is obtain an upper bound for

$$
\left\|U_{++}^{T} \chi_{1}\left(P_{0}\right)\right\|_{L^{2} \rightarrow L^{2}}=\left\|\Pi_{+} U^{T} W(T) \Pi_{+} \chi_{1}\left(P_{0}\right)\right\|, \quad T=T_{E h r} .
$$

We recall that $\Pi_{+}$is the projector associated with the region $\Omega_{+, \alpha}$. Let us indicate that a similar type of dispersion estimate was used by S. Brooks, when studying the delocalization of the eigenstates of quantized hyperbolic automorphisms of the 2-dimensional torus (the so-called "quantum cat maps") Bro10.

To estimate this norm, it will be useful to replace this projector by a smoothed microlocal projector obtained by quantizing a symbol $\chi_{+}=\chi_{+, \alpha} \in S_{1 / 2-\epsilon}^{-\infty}\left(T^{*} X\right)$, such that $\mathrm{Op}_{\hbar}\left(\chi_{+}\right)$"dominates" $\Pi_{+}$:

$$
\Pi_{+} \chi_{1}\left(P_{0}\right)=\mathrm{Op}_{\hbar}\left(\chi_{+}\right) \Pi_{+} \chi_{1}\left(P_{0}\right)+\mathcal{O}\left(\hbar^{\infty}\right)
$$


The norm (3.39) can then be bounded by:

$$
\left\|U_{++}^{T} \chi_{1}\left(P_{0}\right)\right\| \leq \sup _{\left\|u_{1}\right\|=\left\|u_{2}\right\|=1}\left|\left\langle\mathrm{Op}_{\hbar}\left(\chi_{+}\right) u_{2}, U^{T} W(T) \mathrm{Op}_{\hbar}\left(\chi_{+}\right) u_{1}\right\rangle\right|+\mathcal{O}\left(\hbar^{\infty}\right) .
$$

The symbol $\chi_{+}$can be chosen supported inside a set of the form $\Omega_{+, \alpha-C \epsilon} \cap p_{0}^{-1}([1 / 2 \pm$ $\epsilon])$. This set is quite irregular, and the main information we have on it is an estimate on its volume (using large deviation estimates like (3.31)). It is then convenient to use an anti-Wick quantization scheme for $\mathrm{Op}_{\bar{t}}\left(\chi_{+}\right)$, that is use a family of coherent states (Gaussian wavepackets) $\left\{e_{\rho}, \rho \in T^{*} X\right\}$, to define

$$
\mathrm{Op}_{\hbar}\left(\chi_{+}\right) \stackrel{\text { def }}{=} \int \frac{d \rho}{(2 \pi \hbar)^{d}} \chi_{+}(\rho)\left\langle e_{\rho}, \bullet\right\rangle e_{\rho} .
$$

Each coherent state $e_{\rho}$ is normalized, and is microlocalized in a "microscopic ellipse" around $\rho$. This ellipse is chosen to be "adapted" to the flow. Let us describe it using the local Darboux coordinates $\{(y, \eta)\}$ as in 33.3 .2 . The ellipse is "short" in the energy direction, $\Delta \eta_{1} \sim \hbar^{1-\epsilon / 2}$, and "long" in the time direction, $\Delta y_{1} \sim \hbar^{\epsilon / 2}$. The spread along the transverse directions is chosen isotropic: $\Delta y_{j}=\Delta \eta_{j} \sim \hbar^{1 / 2}$, $j=2, \ldots, d$.

The scalar product $\left\langle\mathrm{Op}^{+}\left(\chi_{+}\right) u_{2}, U^{T} W_{s}(T) \mathrm{Op}^{+}\left(\chi_{+}\right) u_{1}\right\rangle$ can now be expressed as a double phase space integral

$$
\iint_{p_{0}^{-1}([1 / 2 \pm \epsilon])} \frac{d \rho_{1} d \rho_{2}}{(2 \pi \hbar)^{2 d}}\left\langle u_{2}, e_{\rho_{2}}\right\rangle\left\langle e_{\rho_{1}}, u_{1}\right\rangle \chi_{+}\left(\rho_{2}\right) \chi_{+}\left(\rho_{1}\right)\left\langle U^{-T / 2} e_{\rho_{2}}, U^{T / 2} W(T) e_{\rho_{1}}\right\rangle .
$$

The state $W(T) e_{\rho_{1}}$ is approximately identical to $e_{\rho_{1}}$. We can then precisely describe the (undamped) evolutions of the coherent states $e_{\rho_{i}}$ up to the times $\pm T / 2$. The state $U^{T / 2} e_{\rho_{1}}$ (resp. $U^{-T / 2} e_{\rho_{2}}$ ) is a (microscopic) Lagrangian state along a leaf of the weak unstable manifold of volume $\sim \hbar^{(d-1+\epsilon) / 2} J_{T / 2}^{+}\left(\rho_{1}\right)$ centered at $\Phi^{T / 2}\left(\rho_{1}\right)$, (resp. a leaf of the weak stable manifold of volume $\sim \hbar^{(d-1+\epsilon) / 2} J_{T / 2}^{+}\left(\Phi^{-T / 2}\left(\rho_{2}\right)\right.$ ) centered at $\left.\Phi^{-T / 2}\left(\rho_{2}\right)\right)$. Using the sharp energy localization of these states and the fact that stable and unstable manifolds intersect transversely to each other, one gets the following bound:

$$
\left|\left\langle U^{-T / 2} e_{\rho_{2}}, U^{T / 2} W(T) e_{\rho_{1}}\right\rangle\right| \leq \frac{\theta\left(\frac{\eta_{1}\left(\rho_{1}\right)-\eta_{1}\left(\rho_{2}\right)}{\hbar^{1-\epsilon}}\right)}{\sqrt{J_{T / 2}^{+}\left(\rho_{1}\right) J_{T / 2}^{+}\left(\Phi^{-T / 2}\left(\rho_{2}\right)\right)}}+\mathcal{O}\left(\hbar^{\infty}\right),
$$

for some $\theta \in C_{c}^{\infty}([-1,1])$. The denominator is bounded below by $e^{\nu_{\min }(d-1) T / 2}$. Inserting this bound in the above double integral and using large deviation estimates similar with (3.31) for the support of $\chi_{+}$, one finally gets (after some manipulations) the bound (3.36).

\section{REFERENCES}

[Anan08] N. Anantharaman, Entropy and the localization of eigenfunctions, Ann. Math. (2) 168, 435-475 (2008)

[Anan10] N. Anantharaman, Spectral deviations for the damped wave equation, GAFA 20 (2010) 593-626

[AN1] N. Anantharaman and S. Nonnenmacher, Half-delocalization of eigenfunctions for the Laplacian on an Anosov manifold, Ann. Inst. Fourier 57(7), 2465-2523 (2007)

\footnotetext{
${ }^{9}$ Because $\chi+$ is supported inside $p_{0}^{-1}([1 / 2-\delta])$,it is sufficient to construct a family of coherent states with $\rho$ in this (compact) region.
} 
[AschLeb] M. Asch and G. Lebeau, The Spectrum of the Damped Wave Operator for a Bounded Domain in $\mathbb{R}^{2}$, Exper. Math. 12 (2003) 227-241

[Bro10] S. Brooks, On the entropy of quantum limits for 2-dimensional cat maps, Commun. Math. Phys. 293 (2010) 231-255

[BuHi07] N. Burq and M. Hitrik, Energy decay for damped wave equations on partially rectangular domains, Math. Res. Lett. 14 (2007) 35-47

[Chris07] H. Christianson, Semiclassical Non-concentration near Hyperbolic Orbits, J. Funct. Anal. 246 (2007) 145-195; Corrigendum, J. Funct. Anal. 258 (2010) 1060-1065

[Chris09] H. Christianson, Applications of Cutoff Resolvent Estimates to the Wave Equation, Math. Res. Lett. Vol. 16 (2009) 577-590

[Chris11] H. Christianson, Quantum Monodromy and Non-concentration Near a Closed Semihyperbolic Orbit, Trans. Amer. Math. Soc. 363 (2011) 3373-3438

[EvZw] C.L. Evans and M. Zworski, Lectures on semiclassical analysis, v.0.75

[Hit03] M.Hitrik, Eigenfrequencies and expansions for damped wave equations, Meth. Appl. Anal. 10 (2003) 1-22

[HitSjo08] M. Hitrik and J. Sjöstrand, Rational invariant tori, phase space tunneling, and spectra for non-selfadjoint operators in dimension 2, Ann. Sci. E.N.S. 41 (2008) 511-571

[HitSjo11] M. Hitrik and J. Sjöstrand, Diophantine tori and Weyl laws for non-selfadjoint operators in dimension two, preprint 2011, arXiv:1102.0889

[HSVN07] M. Hitrik, J. Sjöstrand, and S. Vũ Ngọc, Diophantine tori and spectral asymptotics for non-selfadjoint operators, Amer. J. Math. 129 (2007) 105-182

[KatHas95] A. Katok and B. Hasselblatt, Introduction to the modern theory of dynamical systems, Cambridge UP, 1995

[Kif90] Y. Kifer, Large deviations in dynamical systems and stochastic processes, Trans. Amer. Math. Soc. 321 (1990) 505-524.

[KoTa94] H. Koch and D. Tataru, On the spectrum of hyperbolic semigroups, Comm. Partial Differential Equations, 20, No 5-6, 901-937 (1995)

[Leb93] G.Lebeau, Equation des ondes amorties, Algebraie and geometric methods in mathematical physics, (Kaciveli 1993), 73-109, Math. Phys. Stud. 19, Kluwer Acad. Publ., Dordrecht, 1996

[MarMat84] A.S. Markus and V.I. Matsaev, Comparison theorems for spectra of linear operators, and spectral asymptotics, Trans. Moscow Math. Soc. (1984) 139-187. Russian original in Trudy Moscow. Obshch. 45 (1982), 133-181

[NZ2] S. Nonnenmacher and M. Zworski, Quantum decay rates in chaotic scattering, Acta Math 203 (2009) 149-233

[NZ3] S. Nonnenmacher and M. Zworski, Semiclassical Resolvent Estimates in Chaotic Scattering, Appl. Math. Res. eXpr. 2009, Article ID abp003

[RauTay75] J. Rauch and M. Taylor, Decay of solutions to nondissipative hyperbolic systems on compact manifolds, Commun. Pure Appl. Math. 28 (1975) 501-523

[Ren94] M. Renardy, On the linear stability of hyperbolic PDEs and viscoelastic flows, Zeit. f. angew. Math. Phys. 45 (1994) 854-865

[Riv10] G. Rivière, Entropy of semiclassical measures in dimension 2, Duke Math. J. 155 (2010) $271-335$

[Riv11] G. Rivière, Eigenfunctions of the Laplacian and subsets of small topological pressure, preprint 2011

[Sche10] E. Schenck, Energy decay for the damped wave equation under a pressure condition, Commun. Math. Phys. 300, 375-410 (2010)

[Sche11] E. Schenck, Exponential stabilization without geometric control, preprint 2010

[Sjo00] J. Sjöstrand, Asymptotic distribution of eigenfrequencies for damped wave equations, Publ. Res. Inst. Math. Sci. 36 (2000) 573-611

[SjoZwo07] J. Sjöstrand and M. Zworski, Fractal upper bounds on the density of semiclassical resonances, Duke Math. J. 137 (2007) 381-459

[Zel09] S. Zelditch, Recent developments in mathematical quantum chaos, in Current Developments in Mathematics, 2009, D.Jerison, B.Mazur, T.Mrowka, W.Schmid, R.Stanley, S-T Yau (eds.), International Press 2009 
Institut de Physique théorique, CEA-Saclay, unité de Recherche associée au CNRS, 91191 Gif-Sur-Yvette, France 